
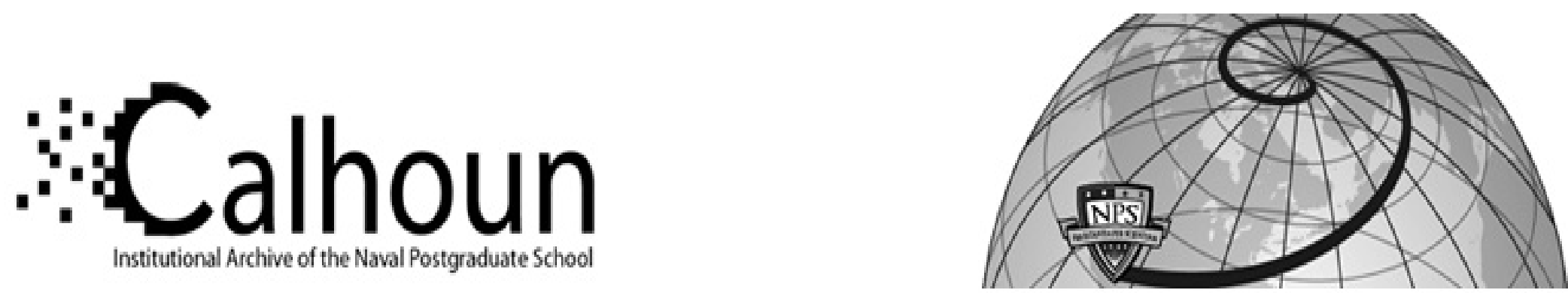

Calhoun: The NPS Institutional Archive DSpace Repository

\title{
Probabilistic stability of an atmospheric model to various amplitude perturbations
}

Melnichenko, Oleg V.; Ivanov, Leonid M.; Margolina, Tatyana M.; Chu, Peter C.

Chu, P.C., L.M., Ivanov, T.M. Margolina, and O.V. Melnichenko, 2002: Probabilistic stability of an atmospheric model to various amplitude perturbations (paper download). Journal of the Atmospheric Sciences, American Meteorological Society, 59, 2860-2873.

https://hdl.handle.net/10945/36091

This publication is a work of the U.S. Government as defined in Title 17, United States Code, Section 101. Copyright protection is not available for this work in the United States.

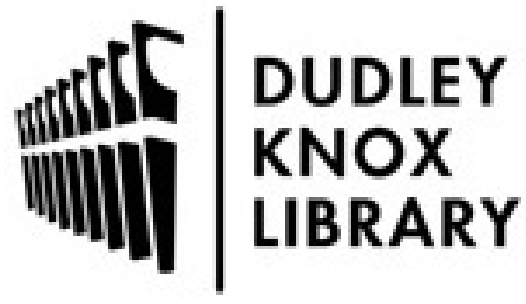

http://www.nps.edu/library
Calhoun is the Naval Postgraduate School's public access digital repository for research materials and institutional publications created by the NPS community. Calhoun is named for Professor of Mathematics Guy K. Calhoun, NPS's first appointed -- and published -- scholarly author.

Dudley Knox Library / Naval Postgraduate School 411 Dyer Road / 1 University Circle Monterey, California USA 93943 


\title{
Probabilistic Stability of an Atmospheric Model to Various Amplitude Perturbations
}

\author{
Peter C. Chu And Leonid M. Ivanov \\ Naval Postgraduate School, Monterey, California \\ Tatyana M. Margolina And Oleg V. Melnichenko \\ Marine Hydrophysical Institute and Center For Ecological Modeling and Recreation Geography, \\ National Academy of Sciences of the Ukraine, Sevastopol, Ukraine
}

(Manuscript received 28 March 2001, in final form 2 April 2002)

\begin{abstract}
Every forecast should include an estimate of its likely accuracy, as a measure of predictability. A new measure, the first passage time (FPT), which is defined as the time period when the model error first exceeds a predetermined criterion (i.e., the tolerance level), is proposed here to estimate model predictability. A theoretical framework is developed to determine the mean and variance of FPT. The low-order Lorenz atmospheric model is taken as an example to show the robustness of using FPT as a quantitative measure for prediction skill. Both linear and nonlinear perspectives of forecast errors are analytically investigated using the self-consistent Nicolis model. The mean and variance of FPT largely depends on the ratio between twice the maximum Lyapunov exponent $(\sigma)$ and the intensity of attractor fluctuations $\left(q^{2}\right), \lambda=2 \sigma / q^{2}$. Two types of predictability are found: $\lambda>1$ referring to low predictability and $\lambda<1$ referring to high predictability. The mean and variance of FPT can be represented by the $e$-folding timescales in the low-predictability range, but not in the high-predictability range. The transition between the two predictability ranges is caused by the variability of the attractor characteristics along the reference trajectory.
\end{abstract}

\section{Introduction}

The following is a commonly asked practical question. How long is an atmospheric (or oceanic) model valid, once it is integrated from its initial state? To answer this question, uncertainty in atmospheric models must be investigated. It is widely recognized that uncertainty can be traced back to three factors (Lorenz 1984a): (a) measurement errors, (b) model errors such as discretization and uncertain model parameters, and (c) chaotic dynamics. Measurement errors cause uncertainty in initial and/or boundary conditions. Discretization not only causes truncation errors but also smallscale "subgrid" processes to be either discarded or parameterized. The chaotic dynamics may trigger a subsequent amplification of small errors through a complex response.

Traditionally, the small amplitude stability analysis (linear error dynamics) is used with the instantaneous error (IE) growth rate and the corresponding $e$-folding timescale as the measures for evaluating the model predictability due to initial condition error (the first kind

Corresponding author address: Dr. Leonid M. Ivanov, Dept. of Oceanography, Code OC/CU, Naval Postgraduate School, Monterey, CA 93943-5000.

E-mail:1mivanov@nps.navv.mil of predictability). The IE growth rate is usually estimated by either the leading (largest) Lyapunov exponent or the amplification factors calculated from the leading singular vectors (SVs; e.g., Lorenz 1984b; Dalcher and Kalnay 1987; Farrell and Ioannou 1996a,b; Vannitsem and Nicolis 1997).

However, the errors may grow to a finite amplitude such as in the medium-range prediction (Vukicevic 1991; Barkmeijer 1996), in the forecast using "imperfect" models (Bofetta et al. 1998; Palmer 2001), and in the models with open boundaries (Gustafsson et al. 1998; Chu 1999; Jiang and Malanotte-Rizzoli 1999). Thus, the linear assumption is no longer applicable and the nonlinear effect should be considered.

Both linear and nonlinear perspectives of forecast errors can be studied using a probabilistic approach based on the analysis of the probability density function (PDF) of the error (e.g., Benzi and Carnavale 1989; Benzi et al. 1999; Nicolis 1992; Ehrendorfer 1994a,b; Molteni and Corti 1998). The given error PDF leads to the full statistical description of the error dynamics. In general, PDF satisfies the Liouville equation which can be solved only by numerical methods even in one-dimensional cases (Ehrendorfer 1994a,b; Nicolis 1992). A practical approach to estimate the PDF of forecast error is to calculate it through ensemble prediction (Leith 1974; Toth and Kalnay 1997). 
To simplify the probabilistic analysis of forecast error dynamics, Ivanov et al. (1994) and Ivanov and Margolina (1999) applied the first passage time (FPT; Gardiner 1985) as a measure of quantitative prediction skill. Here, FPT is defined as the time period when the model forecast error exceeds a predetermined criterion $\varepsilon$ (i.e., the tolerance level) for the first time. In this paper, we plan to show the advantage of using FPT in analyzing the forecast skill of an atmospheric model; to illustrate the capability of such an analysis using a low-order atmospheric model; and to improve the knowledge of how the strength of the initial error and the variations of model parameters affect the model prediction skill.

\section{Measure of predictability}

\section{a. Dynamic constraint}

From a low-order model to a certain level of description such as a global atmospheric spectral model, an $N$ dimensional vector $\mathbf{x}(t)=\left[x^{(1)}(t), x^{(2)}(t), \ldots, x^{(N)}(t)\right]$ is used to represent the full set of variables characterizing the dynamics of the atmosphere. Let the dynamic law be given by:

$$
\frac{d \mathbf{x}}{d t}=\mathbf{f}(\mathbf{x}, t)
$$

where $\mathbf{f}$ is a functional including nonlinear interactions between different scales, a parameterization of subgrid scales and forcing. The aim of individual (oceanic or atmospheric) prediction is to find the solution of (2.1) with an initial condition

$$
\mathbf{x}\left(t_{0}\right)=\mathbf{x}_{0} .
$$

Uncertainty in atmospheric (or oceanic) models is caused by measurement errors (initial and/or boundary condition errors), model discretization, and uncertain model parameters. This leads to the addition of stochastic forcing into atmospheric (oceanic) models. Moreover, stochastic forcing may also be added to the forecast model in the parameterization of nonlinear interactions (Penland and Sardeshmukn 1995; Leith 1996; Palmer 2001).

For simplicity, the stochastic forcing $\left(\mathbf{f}^{\prime}\right)$ is assumed to be multiplicative or additive white noise, and (2.1) becomes

$$
\begin{aligned}
\frac{d \mathbf{x}}{d t} & =\mathbf{f}(\mathbf{x}, t)+\mathbf{f}^{\prime}(\mathbf{x}, t), \\
\mathbf{f}^{\prime}(\mathbf{x}, t) & =\mathbf{k}(\mathbf{x}, t) \mathbf{g}(t),
\end{aligned}
$$

where $\mathbf{k}(\mathbf{x}, t)$ and $\mathbf{g}(t)$ are the forcing covariance matrix $\left\{\mathbf{k}_{i j}\right\}$ (dimension of $\left.N \times N\right)$ and the vector delta-correlated process (dimension of $N$ ), respectively.

\section{b. FPT for forecast error}

Let $\hat{\mathbf{x}}(t)$ be the reference solution which satisfies (2.2) with the initial condition $\mathbf{x}\left(t_{0}\right)$. The forecast error $\mathbf{z}$ is determined as

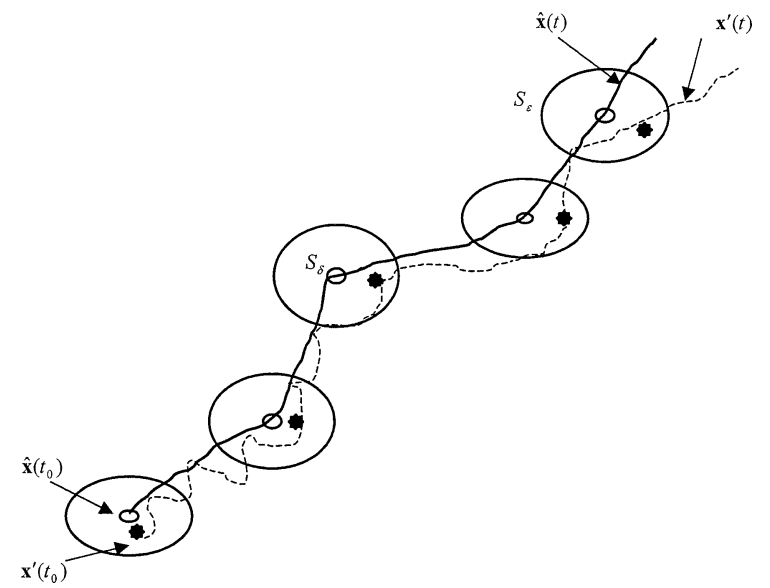

FIG. 1. Phase space trajectories of the reference solution $\hat{\mathbf{x}}$ (solid curve), one of the ensemble realizations $\mathbf{x}^{\prime}$, and ellipsoids $S_{\varepsilon}(t)$ and $S_{\delta}(t)$ centered at $\hat{\mathbf{x}}$. The positions of $\hat{\mathbf{x}}$ and $\mathbf{x}^{\prime}$ at time instance are denoted by “*” and " $\bigcirc$ ", respectively. FPT is represented by a time at which perturbed trajectory $\mathbf{x}^{\prime}$ leaves the ellipsoid $S_{\varepsilon}(t)$ at the first time.

$$
\mathbf{z}(t)=\hat{\mathbf{x}}(t)-\mathbf{x}^{\prime}(t),
$$

where $\mathbf{x}^{\prime}(t)$ is an individual prediction corresponding to perturbing initial condition and/or forcing $\mathbf{f}+\mathbf{f}^{\prime}$. The rmse is used to estimate the model forecast skill:

$$
J=\left\langle\mathbf{z}^{\mathrm{T}} \mathbf{A} \mathbf{z}\right\rangle,
$$

where $\mathbf{A}$ is the weight matrix, the superscript $\mathrm{T}$ denotes the transpose operator, the bracket represents the ensemble average over realizations generated by stochastic forcing, uncertain initial conditions, and uncertain model parameters in (2.2).

To quantify FPT, we first define two model error limits. First, the forecast error cannot be less than a minimum-scale $\delta$, which depends on the level of thermal and intrinsic noises existing in the model. In general, this scale functionally depends on the square root of the subgrid-scale kinetic energy $E^{1 / 2}$. The simplest parameterization is $\delta$ proportional to $E^{1 / 2}$, with the proportionality depending on the selection of the subgrid-scale parameterization (Aurell et al. 1996). Second, the forecast error cannot be more than a maximum-scale (tolerance level) $\varepsilon$.

The ratio between the maximum and minimum limits is large

$$
z_{1}=\varepsilon / \delta \gg 1,
$$

in atmospheric circulation models. Two ellipsoids $S_{\varepsilon}$ and $S_{\delta}$ are defined by $J=\varepsilon^{2}$ and $J=\delta^{2}$, with the center at $\hat{\mathbf{x}}(t)$ (Fig. 1). The model rmse $(J)$ is bounded by the two limits

$$
\delta^{2} \leq J \leq \varepsilon^{2} .
$$

FPT is a stochastic time period $\tau$ for which $\mathbf{x}^{\prime}(t)$ leaves a domain limited by $S_{\varepsilon}$ for the first time. From physical point of view, it determines the low limit of the pre- 
dictability time because we neglect the impact of restoration of model prediction skill a priori, which occurs in large atmospheric models (Anderson and van der Dool 1994).

To calculate the moments of FPT, the probability density of prediction (PDP) is $P\left(t_{0}, \mathbf{z}_{0}, t-t_{0}\right)$, which is the probability of the time period $t-t_{0}$ that the forecast error is less than $\varepsilon$ when the initial error is $\mathbf{z}_{0}$ (Ivanov and Margolina 1999). Thus, the stability analysis on the reference solution $\hat{\mathbf{x}}$ (Fig. 1) using FPT should be classified as the probabilistic (stochastic) stability analysis (Has'minskii 1980).

Use of FPT and PDP has the following advantages in evaluating model predictability skill. First, FPT is a measure of model forecast skill for both linear and nonlinear error regimes. Second, the moments of FPT characterize high-order variability of the prediction skill. This variability is determined only from the knowledge of a single-point distribution function, PDP. Third, PDP and naturally, the moments of FPT, can be effectively calculated by ensemble prediction techniques. Such a calculation consumes less computer resources because PDP is determined only inside the ellipsoid $S_{\varepsilon}$, rather than in the whole phase space. Fourth, an iterative technique (Ivanov et al. 1999) and analytical approach (Chu et al. 2002) are developed to find the structure of PDP. Analytical estimate of PDP is possible even for multidimensional error models.

\section{Pontryagin-Kolmogorov equation}

The temporal evolution of PDP, for the model (2.2), satisfies the Pontryagin-Kolmogorov equation (PKE; Pontryagin et al. 1969) with the first-order partial differentiation with respect to time, and the second-order partial differentiations with respect to initial condition errors,

$$
\begin{gathered}
\frac{\partial P}{\partial t}-\left(f_{i}-\frac{d \hat{x}_{i}}{d t}\right) \frac{\partial P}{\partial z_{i}^{0}}-\frac{1}{2} k_{i l} k_{l j} \frac{\partial^{2} P}{\partial z_{i}^{0} z_{j}^{0}}=0, \\
i, j, l=1, \ldots, N,
\end{gathered}
$$

where the coefficients $k_{i j}$ are the components of the forcing covariance matrix $\boldsymbol{\kappa}(\mathbf{x}, t)$ and $\left(z_{1}^{0}, z_{2}^{0}, \ldots z_{N}^{0}\right)$ are the components of the initial error $\mathbf{z}_{0}$.

One initial condition and two boundary conditions (with respect to $\mathbf{z}_{0}$ ) are needed to solve (3.1). The initial condition of (3.1) is given by

$$
P\left(t_{0}, \mathbf{z}_{0}, 0\right)=1 \text {, }
$$

which indicates that the vector of initial error $\mathbf{z}_{0}$ is always less than the given tolerance.

The model error vector $\mathbf{z}$ is bounded by two limits: minimum error limit (noise level $\delta$ ) and maximum error limit (tolerance level $\varepsilon$ ). If the initial error vector $\mathbf{z}_{0}$ reaches the tolerance level [i.e., $\mathbf{z}_{0}$ hits the boundary of $\left.S_{\varepsilon}\left(t_{0}\right)\right]$, the model loses prediction capability at the first place:

$$
P\left(t_{0}, \mathbf{z}_{0}, t-t_{0}\right)=0 \quad \text { at } J=\varepsilon^{2},
$$

which is called the absorbing type boundary condition. It is clear that the forecast error can not be less than $\delta$, which leads to

$$
k_{i j} \frac{\partial P}{\partial z_{j}^{0}}=0 \quad \text { at } J=\delta^{2},
$$

which is called the reflecting boundary condition. In general, the reflecting and absorbing boundary conditions can be simultaneously applied on $S_{\varepsilon}$ and $S_{\delta}$.

The $k$ th moment $(k=1,2, \ldots)$ of FPT is calculated using PDP by

$$
\begin{gathered}
\tau_{k}\left(\mathbf{z}_{0}\right)=k \int_{t_{0}}^{\infty} P\left(t_{0}, \mathbf{z}_{0}, t-t_{0}\right)\left(t-t_{0}\right)^{k-1} d t, \\
k=1, \ldots, \infty .
\end{gathered}
$$

The mean and variance of FPT can be calculated from the first two moments:

$$
\begin{aligned}
\langle\tau\rangle & =\tau_{1}, \\
\left\langle\delta \tau^{2}\right\rangle & =\tau_{2}-\tau_{1}^{2},
\end{aligned}
$$

where the bracket denotes the ensemble average over realizations generated by stochastic forcing. The mean and variance of FPT are both local characteristics of the prediction skill since they depend on position $\mathbf{z}_{0}$ on the reference trajectory $\hat{\mathbf{x}}$.

The prediction skill of atmospheric models is currently verified using the perfect model concept with uncertainty appearing only in the initial conditions. Such an uncertainty is easily taken into account in our approach through additional averaging of (3.6) and (3.7) with respect to an ensemble of initial perturbations.

\section{A low-order atmospheric model}

Determination of prediction skill of a low-order atmospheric model proposed by Lorenz (1984a) is taken as an example to demonstrate the usefulness of our approach. The Lorenz system is the simplest possible model capable of representing an unmodified or modified Hadley circulation, determining its stability and, if it is unstable, representing a stationary or migratory disturbance. The model consists of the three ordinary nondimensional differential equations:

$$
\begin{aligned}
& \frac{d x_{1}}{d t}=-x_{2}^{2}-x_{3}^{2}-a x_{1}+a F, \\
& \frac{d x_{2}}{d t}=x_{1} x_{2}-b x_{1} x_{3}-x_{2}+G, \\
& \frac{d x_{3}}{d t}=b x_{1} x_{2}+x_{1} x_{3}-x_{3},
\end{aligned}
$$

where $t$ is the time scaled by five days; $x_{1}$ represents 
the intensity of the symmetric globe-encircling westerly wind, and also the poleward temperature gradient which is assumed to be in permanent equilibrium with the wind; $x_{2}$ and $x_{3}$ are the cosine and sine phases, respectively, of a chain of superposed large-scale eddies, which transport heat at a rate proportional to the square of their amplitude, and transport no angular momentum at all. The terms $a F$ and $G$ represent symmetric and asymmetric thermal forcing. The terms $x_{1} x_{2}$ and $x_{1} x_{3}$ describe amplification of eddies through interaction with the westerly currents. The displacement of eddies by the westerly wind is parameterized using the terms $-b x_{1} x_{3}$ and $b x_{1} x_{2}$. The parameter $a$ is the damping coefficient.

The traditional stability analysis on the Lorenz model (4.1) leads to three Lyapunov exponents. Among them, only one is positive. Projection of the three-dimensional forecast error vector onto the unstable manifold leads to a self-consistent model (Nicolis 1992)

$$
\begin{aligned}
\frac{d \xi}{d t} & =(\sigma-g \xi) \xi+v(t) \xi,\left.\quad \xi\right|_{t=t_{0}}=\xi_{0}, \\
\xi & \in[0, \infty),
\end{aligned}
$$

where $\xi$ is the nondimensional amplitude of error, and $g$ is a nonnegative, generally time-independent nonlinear parameter whose properties depend on the underlying attractor. Equation (4.2) is written in Ito form. The tangent approximation of error growth leads to $g=0$. The eigenvector $\xi$ is associated with the positive Lyapunov exponent $(\sigma)$. The term $v(t) \xi$ is a specially chosen stochastic forcing with zero mean and pulse-type variance

$$
\langle v(t)\rangle=0,\left\langle v(t) v\left(t^{\prime}\right)\right\rangle=q^{2} \Delta\left(t-t^{\prime}\right),
$$

where the bracket is defined as the ensemble mean over realizations generated by the stochastic forcing, $\Delta$ is the Delta function, and $q^{2}$ is the intensity of attractor fluctuations modeled as multiplicative noise forcing.

The combination of the free model parameters $\sigma, g$, and $q^{2}$ affects the model prediction skill. Nicolis (1992) used the following values:

$$
\sigma=0.64, \quad g=0.3, \quad q^{2}=0.2 .
$$

In reality the dynamical characteristics of the atmosphere vary considerably between different synoptic situations. Model forecast skill depends on various factors such as season, location, and boundary conditions. Evidence shows variability of forecast skill in operational models such as the European Centre for Medium-Range Weather Forecasts (ECMWF) model. Therefore, the parameters $\sigma, g$, and $q^{2}$ should be time and attractor dependent. This problem is not addressed here. However, in contrast to Nicolis (1992) we assume

$$
\begin{aligned}
0.2 & \leq \sigma \leq 0.64, \quad g=0.3, \\
0.01 & \leq q^{2} \leq 0.6
\end{aligned}
$$

in this study. Since the Nicolis model is analytical, the parameter $\delta$ should be small and $z_{1} \equiv \varepsilon / \delta=10^{5}$ is assumed. This should be satisfactory for the quality analysis of the prediction skill.

Use of the self-consistent model (4.2) has several explicit advantages. First, it allows analytical study on linear and nonlinear perspective of forecast error. Second, the methodology developed by Nicolis (1992) can be generalized to more realistic atmospheric models. Third, the model contains several dynamical regimes of forecast error behavior. Obviously, their analytical study may be useful for the interpretation of results obtained by large atmospheric models.

\section{Mean and variance of FPT}

For the self-consistent error model (4.2), the mean and variance of FPT can be computed without the determination of PDP. PDP for the Nicolis model (4.2) satisfies the following PKE (Pontryagin et al. 1969; Gardiner 1985; Ivanov et al. 1994):

$$
\frac{\partial P}{\partial t}-\left(\sigma-g \xi_{0}\right) \xi_{0} \frac{\partial P}{\partial \xi_{0}}-\frac{1}{2} q^{2} \xi_{0}^{2} \frac{\partial^{2} P}{\partial \xi_{0}^{2}}=0,
$$

with the initial condition

$$
P\left(t_{0}, \xi_{0}, 0\right)=1,
$$

and boundary conditions

$$
\begin{gathered}
\left.P\left(t_{0}, \xi_{0}, t-t_{0}\right)\right|_{\xi_{0}=\varepsilon}=0 \\
\left.\frac{\partial P\left(t_{0}, \xi_{0}, t-t_{0}\right)}{\partial \xi_{0}}\right|_{\xi_{0}=\delta}=0 .
\end{gathered}
$$

Multiplying (5.1) by $\left(t-t_{0}\right)^{k}$ and then integrating with time from $t_{0}$ to $\infty$, we obtain a set of equations for the moments $\tau_{k}(k=1,2, \ldots)$ :

$$
\begin{aligned}
\left(\sigma \xi_{0}-g \xi_{0}^{2}\right) \frac{d \tau_{k}}{d \xi_{0}}+\frac{q^{2} \xi_{0}^{2}}{2} \frac{d^{2} \tau_{k}}{d \xi_{0}^{2}} & =-k \tau_{k-1}, \\
\delta & \leq \xi_{0} \leq \varepsilon .
\end{aligned}
$$

The boundary conditions for $\tau_{k}$ also are obtained through integrating (5.3) with time

$$
\left.\tau_{k}\right|_{\xi_{0}=\varepsilon}=0,\left.\quad \frac{d \tau_{k}}{d \xi_{0}}\right|_{\xi_{0}=\delta}=0
$$

The mean FPT is the same as the first moment $\tau_{1}$, and the variance of FPT is computed from the first and second moments [see (3.7)]. Simple mathematical manipulations give the following equations:

$$
\begin{gathered}
\left(\sigma \xi_{0}-g \xi_{0}^{2}\right) \frac{d\langle\tau\rangle}{d \xi_{0}}+\frac{q^{2} \xi_{0}^{2}}{2} \frac{d^{2}\langle\tau\rangle}{d \xi_{0}^{2}}=-1 \\
\left(\sigma \xi_{0}-g \xi_{0}^{2}\right) \frac{d\left\langle\delta \tau^{2}\right\rangle}{d \xi_{0}}+\frac{q^{2} \xi_{0}^{2}}{2} \frac{d^{2}\left\langle\delta \tau^{2}\right\rangle}{d \xi_{0}^{2}}=-q^{2} \xi_{0}^{2}\left(\frac{d\langle\tau\rangle}{d \xi_{0}}\right)^{2} .
\end{gathered}
$$



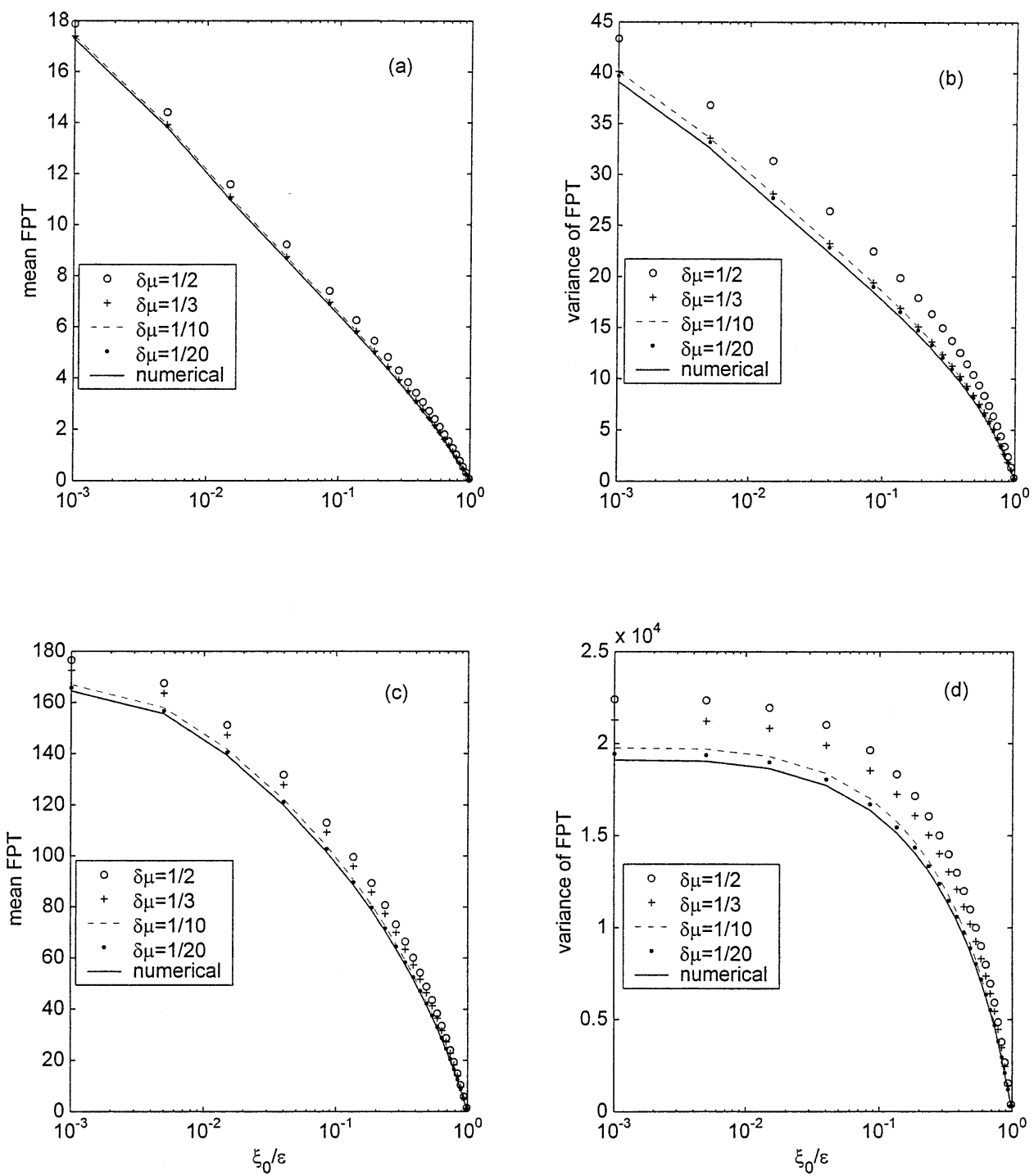

Fig. 2. Comparison of prediction skill between iterative (with different iteration parameter $\Delta \mu$ ) and numerical solutions for (a) mean FPT with $\lambda=6.4$, (b) variance of FPT with $\lambda=6.4$, (c) mean FPT with $\lambda=1$, and (d) variance of FPT with $\lambda=1$.

\section{Iterative solutions}

An iterative method (Ivanov et al. 1999), whose mathematical detail is listed in appendix $\mathrm{A}$, is proposed to solve (5.6) and (5.7). The iteration parameter $(\Delta \mu)$ is defined as the inverse of the number of iteration $(I)$; that is, $\Delta \mu=1 / I$.

It is clear that the mean and variance of FPT, as well as the iteration parameter depend on the model parameters $(\sigma$ and $q)$ and the tolerance level. To illustrate how fast the convergence of the iterative process is, the iterative solutions $\left(\langle\tau\rangle,\left\langle\delta \tau^{2}\right\rangle\right)$ are compared with the numerical solutions $\left(\langle\tau\rangle_{\text {num }},\left\langle\delta \tau^{2}\right\rangle_{\text {num }}\right)$, obtained using the finite difference method with the second-order accuracy (Press et al. 1992). Two sets of calculations are conducted (Fig. 2) with the model parameters. (a) In case
$1, g=0.3, \sigma=0.64, q^{2}=0.4(\lambda=3.2)$, and $\varepsilon=0.1$. (b) In case 2, $g=0.3, \sigma=0.2, q^{2}=0.4(\lambda=1)$, and $\varepsilon=0.5$. Here, $\lambda=2 \sigma / q^{2}$.

The root-mean-square errors between iterative and numerical solutions,

$$
\begin{aligned}
& D_{1}=\operatorname{rmse}\left(\frac{\langle\tau\rangle-\langle\tau\rangle_{\mathrm{num}}}{\langle\tau\rangle_{\mathrm{num}}}\right), \\
& D_{2}=\operatorname{rmse}\left(\frac{\left\langle\delta \tau^{2}\right\rangle-\left\langle\delta \tau^{2}\right\rangle_{\mathrm{num}}}{\left\langle\delta \tau^{2}\right\rangle_{\mathrm{num}}}\right),
\end{aligned}
$$

are used to represent the accuracy of the iteration procedure. For case $1, D_{1}=0.85 \%$ and $D_{2}=5.6 \%$ with the iteration parameter $\Delta \mu=1 / 2$; and $D_{1}=0.03 \%$ and 
$D_{2}=0.25 \%$ with the iteration parameter $\Delta \mu=1 / 3$ (Figs. 2a,b). For case $2, D_{1}=1.7 \%$ and $D_{2}=5.2 \%$ with the iteration parameter $\Delta \mu=1 / 2$; and $D_{1}=0.8 \%$ and $D_{2}$ $=2.2 \%$ with the iteration parameter $\Delta \mu=1 / 3$ (Figs. $2 \mathrm{c}, \mathrm{d})$. Thus, with the iteration parameter $\Delta \mu=1 / 3$, the iterative solutions are quite close to the numerical solutions, and therefore, can be used for evaluating the model prediction skill.

\section{Characteristics of prediction skill}

The model prediction skill $(S)$ that is represented by the mean and variance of FPT strongly depends on the model parameters such as $\lambda$ in the Nicolis model. If a critical value $\lambda_{0}$ is found where $\mathrm{d} S / \mathrm{d} \lambda$ is discontinuous, $S$ may behave differently between $\lambda>$ $\lambda_{0}$ and $\lambda<\lambda_{0}$. Low (high) $S$ values in one side of $\lambda_{0}$ is referred to low (high) predictability. Low predictability (dynamical chaos) may coexist with high predictability (dynamical behavior) even for the same attractor (Ziehmann et al. 2000). In the Nicolis system, low predictability is found for $\lambda>1$, and high predictability is found for $\lambda<1$.

\section{a. Low predictability $(\lambda>1)$}

Low predictability is characterized by low values of mean and variance of FPT, high sensitivity to initial condition errors, and usually occurs in chaotic dynamics. This case has been numerically studied by Nicolis (1992) for $\lambda=6.4$.

\section{1) LINEAR REGIME}

For the linear regime $(g=0)$, the mean and variance of FPT have the analytical expressions

$$
\begin{aligned}
\langle\tau\rangle^{[0]} & =\frac{2}{q^{2}(\lambda-1)}\left[\ln (z)-\frac{1}{\lambda-1} z_{1}^{-(\lambda-1)}\left(z^{\lambda-1}-1\right)\right], \\
z & =\frac{\varepsilon}{\xi_{0}}, \quad z_{1}=\frac{\varepsilon}{\delta} .
\end{aligned}
$$

Since $z_{1} \gg 1$ and $\lambda>1$, Eq. (7.1) can be rewritten by

$$
\langle\tau\rangle^{[0]} \approx \frac{2}{\left(2 \sigma-q^{2}\right)} \ln (z),
$$

which is the classical $e$-folding time. Forecast accuracy weakly depends on $\delta$ and, naturally, on the model subgrid-scale parameterizations. Furthermore, $\langle\tau\rangle^{[0]}$ is bounded if $\delta$ is nonzero.

Equation (7.2) further shows that $\langle\tau\rangle^{[0]}$ increases with increasing intensity of attractor fluctuations $q^{2}$. This is consistent with the Benzi and Carnevale (1989) result obtained for the tangent model (4.2) that the most probable growth of forecasting error is depressed as the attractor fluctuations increase.

Similar to (7.1), the variance of FPT is calculated by

$$
\left\langle\delta \tau^{2}\right\rangle^{[0]} \approx \frac{8}{q^{4}(\lambda-1)^{3}} \ln (z),
$$

which indicates that the variance of FPT also has an $e$ folding dependence on $z$. However, the generality of this analytical result (i.e., $e$-folding variance of FPT) into operational atmospheric models should be further investigated. The mean and variance of FPT are calculated with three different tolerance levels $(\varepsilon=0.02,0.2$, and 2 ) and various values of initial error $\xi_{0}$. Both mean and variance of FPT reveal $e$-folding type with $z$ when $\xi_{0}$ $\leq 0.5$ (Fig. 3)

\section{2) Nonlinear REgime}

For the nonlinear regime $(g \neq 0)$, the mean FPT is obtained using the iterative method illustrated in appendix A:

$$
\langle\tau\rangle \approx\langle\tau\rangle^{[0]}+\frac{4}{3 q^{2}(\lambda-1)}\left(R^{[1]}+R^{[2]}+R^{[3]}+R^{[4]}\right),
$$

where the functions $R^{[1]}, R^{[2]}, R^{[3]}$, and $R^{[4]}$ are determined as

$$
\begin{aligned}
& R^{[1]}=A_{1} \frac{g \varepsilon}{q^{2}}\left(1-z^{-1}\right), \quad R^{[2]}=A_{2} \frac{g^{2} \varepsilon^{2}}{q^{4}}\left(1-z^{-2}\right), \\
& R^{[3]}=A_{3} \frac{g^{3} \varepsilon^{3}}{q^{6}}\left(1-z^{-3}\right), \quad \text { and } \\
& R^{[4]}=A_{4} \frac{g^{4} \varepsilon^{4}}{q^{8}}\left(1-z^{-4}\right),
\end{aligned}
$$

with the coefficients

$$
\begin{aligned}
& A_{1}=\frac{5}{\lambda}, \quad A_{2}=\frac{16}{\lambda(\lambda+1)}, \\
& A_{3}=\frac{5.9}{\lambda(\lambda+1)(\lambda+2)}, \quad \text { and } \\
& A_{4}=\frac{4.7}{\lambda(\lambda+1)(\lambda+2)(\lambda+3)} .
\end{aligned}
$$

As an example, for $\lambda=3.2$, the coefficients are:

$$
\begin{aligned}
& A_{1}=1.5626, \quad A_{2}=0.678, \quad A_{3}=0.030, \\
& A_{4}=0.002,
\end{aligned}
$$

which indicates a fast convergence of the iteration process (fast reduction values from $A_{1}$ to $A_{4}$ ). If $\lambda=$ 6.4 [the parameters take on the same values as in Nicolis (1992)], the convergence of the iteration is even faster.

The expression for the variance of FPT is quite cumbersome, and is not listed here. Figure 3 shows the comparison of the mean and variance of FPT between linear and nonlinear regimes with three different tolerance levels ( $\varepsilon=0.02,0.2$, and 2 ) and $\lambda=3.2$. For small tolerance levels $(\varepsilon=0.02,0.2)$, there is almost 

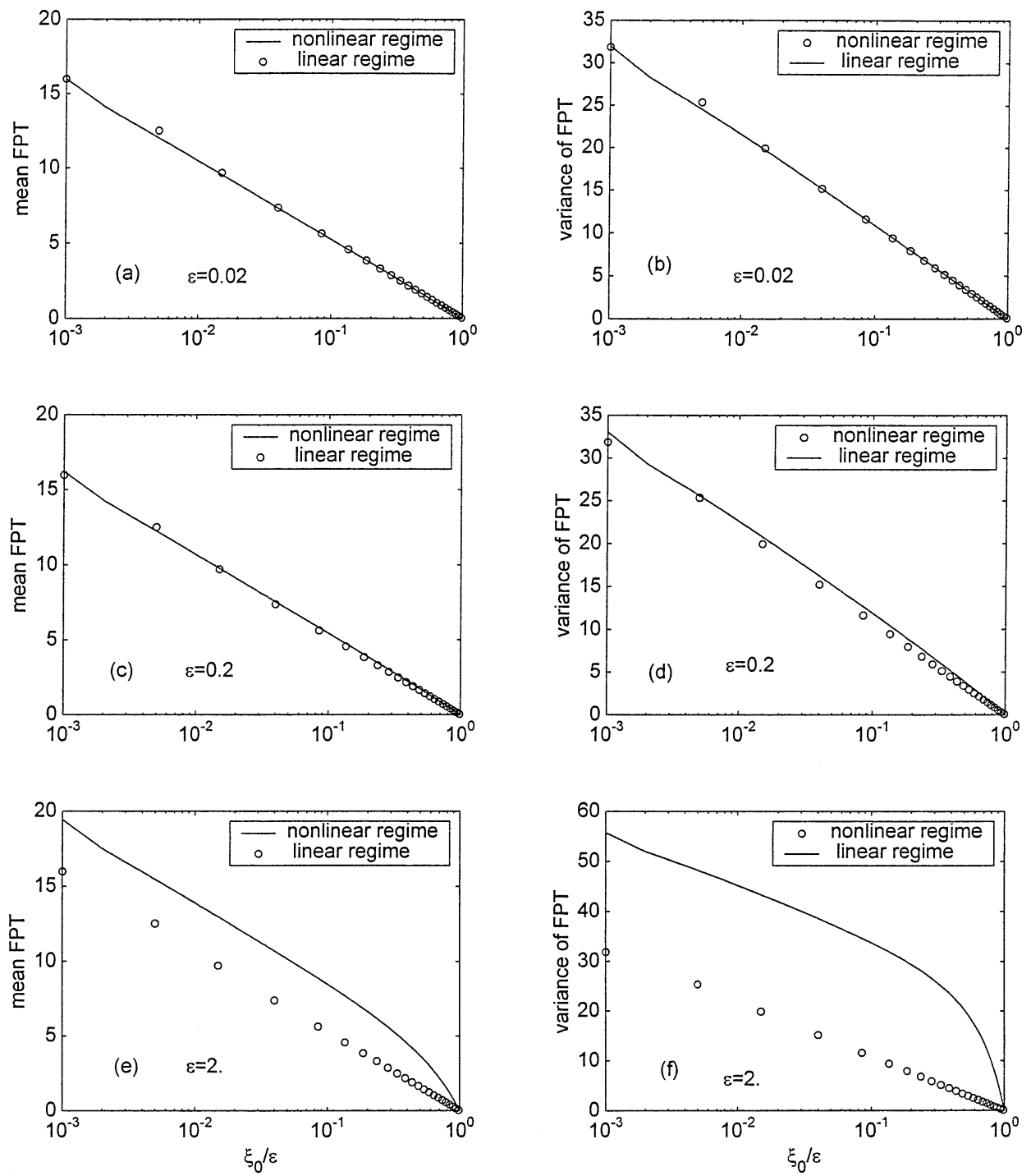

FIG. 3. Comparison of prediction skill between linear and nonlinear regimes for (a) mean FPT with $\varepsilon$ $=0.02$, (b) variance of FPT with $\varepsilon=0.02$, (c) mean FPT with $\varepsilon=0.2$, (d) variance of FPT with $\varepsilon=$ 0.2 , (e) mean FPT with $\varepsilon=2$, and (f) variance of FPT with $\varepsilon=2$.

no difference between linear and nonlinear regimes. The difference increases with the tolerance level. As the tolerance level increases to $\varepsilon=2$, the difference becomes evident.

For a small initial error $\left(\xi_{0} \ll 1\right)$, (7.4) becomes

$$
\begin{aligned}
\langle\tau\rangle \approx \frac{2}{q^{2}(\lambda-1)}( & \ln (z)+\frac{10}{3 \lambda} g \varepsilon \\
& \left.+\frac{32}{3 \lambda(\lambda+1)} \frac{g^{2} \varepsilon^{2}}{q^{2}}+\cdots\right),
\end{aligned}
$$

which indicates that the increase of the mean FPT with increasing $q^{2}$; that is to say, the mean FPT, is larger for the nonlinear regime than for the linear regime.
For a large initial error $\xi_{0}$, the dependence of the high-order iterations on the stochastic forcing $\left(q^{2}\right)$ is complicated. For example, the impact of the third iteration is given by

$$
\begin{aligned}
U & =\frac{4}{3 q^{2}(\lambda-1)} R^{[4]} \\
& =\frac{18.8 g^{4} \varepsilon^{4}}{3 q^{10}(\lambda-1) \lambda(\lambda+1)(\lambda+2)(\lambda+3)}\left(1-z^{-4}\right) .
\end{aligned}
$$

Three $U$ values (for $q^{2}=0.01,0.02$, and 0.2 ), and in turn the two ratios, are computed:

$$
\begin{aligned}
& U\left(q^{2}=0.01\right) / U\left(q^{2}=0.02\right)=1.04, \\
& U\left(q^{2}=0.02\right) / U\left(q^{2}=0.2\right)=0.53,
\end{aligned}
$$



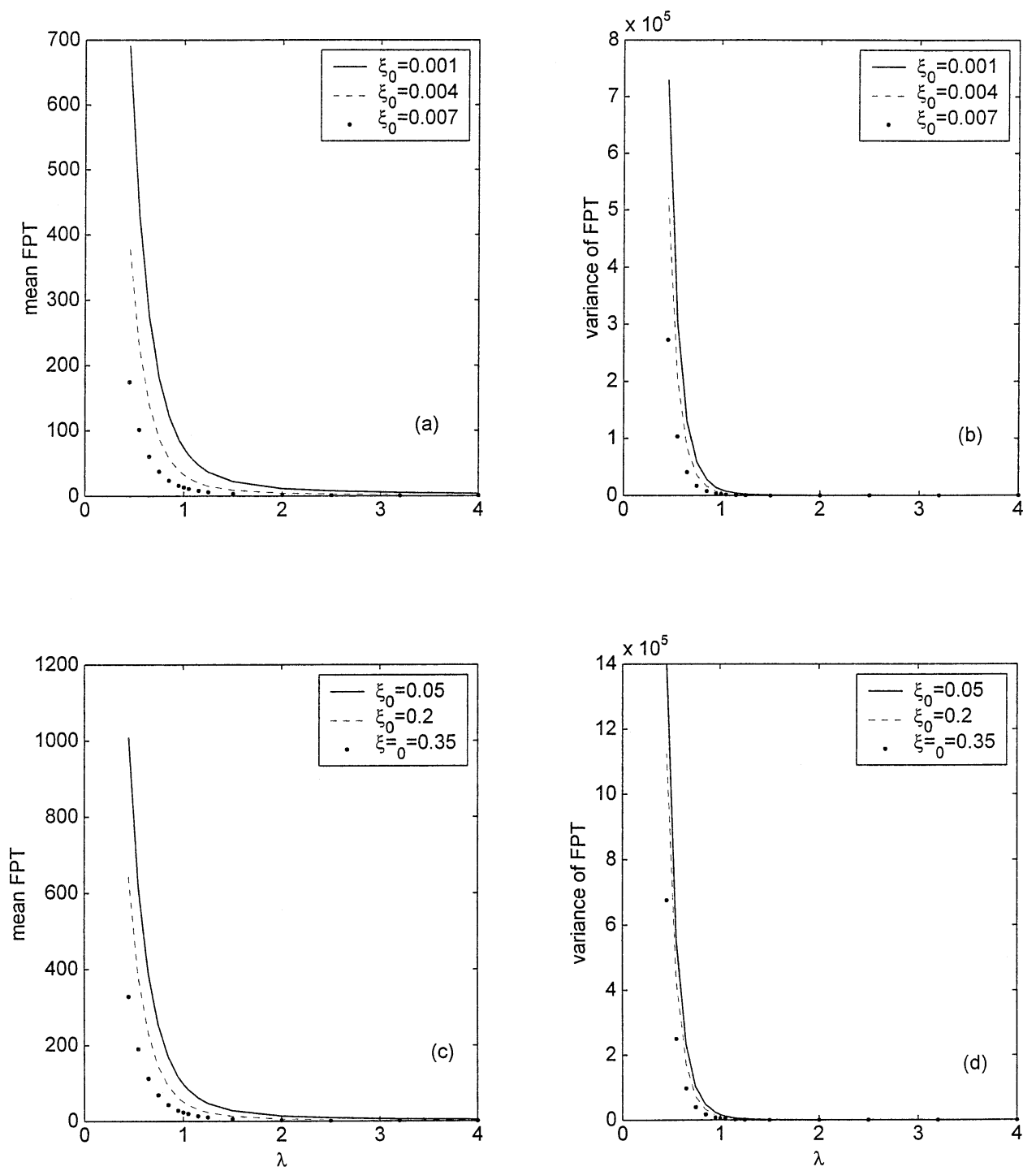

FIG. 4. Dependence of the prediction skill on $\lambda$ for (a) mean FPT with $\varepsilon=0.1$, (b) variance of FPT with $\varepsilon=0.1$, (c) mean FPT with $\varepsilon=0.5$, and (d) variance of FPT with $\varepsilon=0.5$.

which indicates that $U$ is reduced when $q^{2}$ increases slightly from $q^{2}=0.01$ to $q^{2}=0.02$; and then is increased when $q^{2}$ increases drastically from $q^{2}=0.02$ to $q^{2}=0.2$.

\section{b. Transition to high predictability $(\lambda=1)$}

The point $\lambda=1$ is a threshold between two kinds of prediction skill. Here, the mean and variance of FPT cannot be described by the $e$-folding type dependence on $z$ even for infinitesimal and small initial errors. They depend on the noise level $\delta$. To show a change of the model predictability at $\lambda=1$, the mean and variance of FPT are calculated using (5.6) and (5.7) with various tolerance levels and initial errors for linear and nonlinear regimes.

The two different predictability ranges (low and high) can be identified from the dependence of mean and variance of FPT on $\lambda$ value (Fig. 4). Notice that the slope of curves and functional dependences for the mean and variance of FPT have a drastic change at around $\lambda=$ 1 , that is, the low-predictability range (low mean and large variance of FPT) to high-predictability range (high mean and large variance of FPT).

\section{1) LinEAR REgime}

For the linear regime $(g=0)$, the mean and variance of FPT are given by 

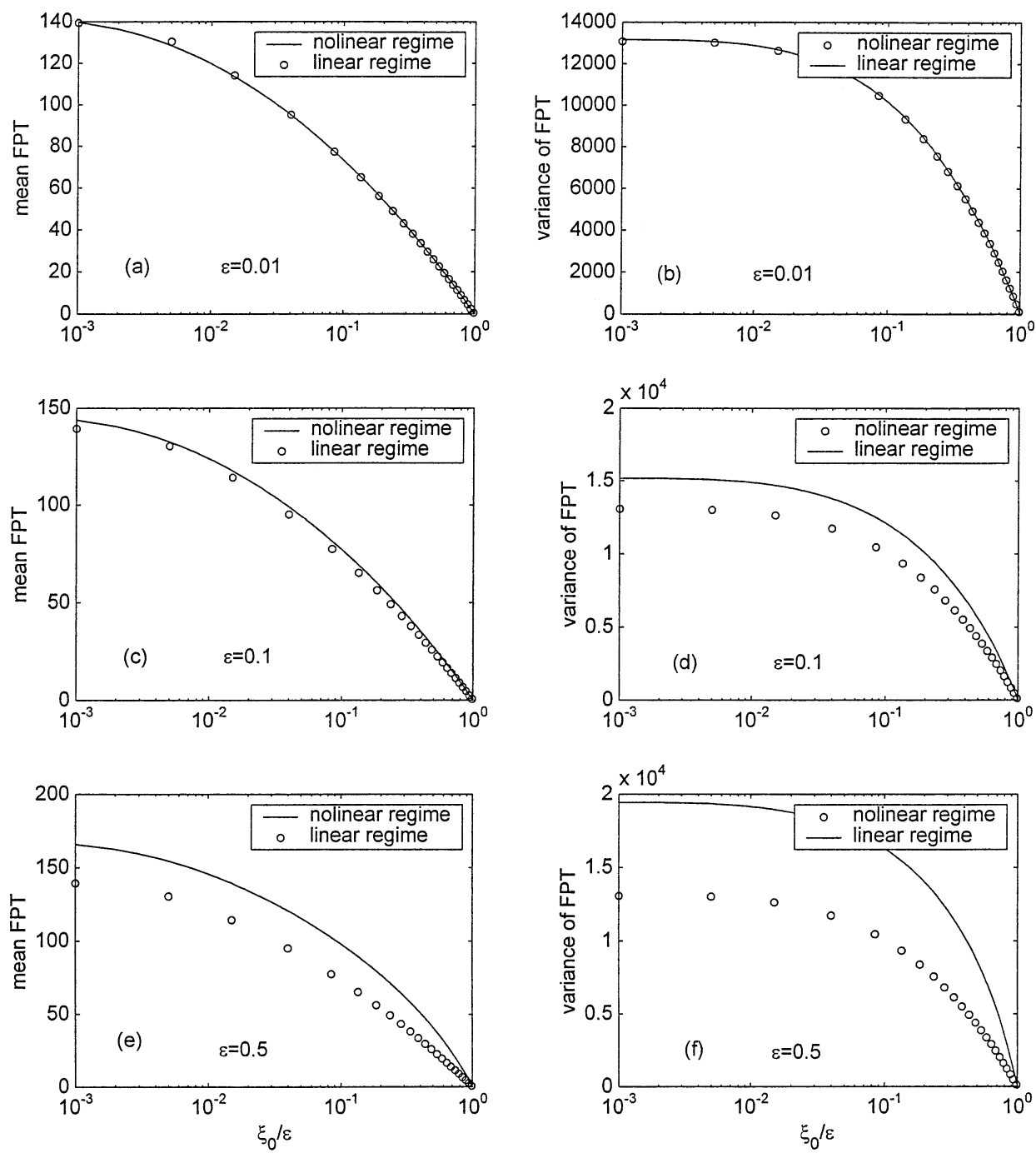

FIG. 5. Dependence of prediction skill on $\xi_{0} / \varepsilon$ for linear and nonlinear regimes with $\lambda=1$ (transition between low and high predictability) for (a) mean FPT with $\varepsilon=0.01$, (b) variance of FPT with $\varepsilon=0.01$, (c) mean FPT with $\varepsilon=0.1$, (d) variance of FPT with $\varepsilon=0.1$, (e) mean FPT with $\varepsilon=0.5$, and (f) variance of FPT with $\varepsilon=0.5$.

$$
\begin{aligned}
\langle\tau\rangle^{[0]} & =\frac{1}{q^{2}}\left[\left(\lambda_{1}\right)^{2}-\left(\lambda_{2}\right)^{2}\right] \\
& =\frac{1}{q^{2}} \ln \left(\frac{\varepsilon}{z_{0}}\right)\left[\ln \left(\varepsilon z_{0}\right)-\ln \delta^{2}\right], \\
\left\langle\delta \tau^{2}\right\rangle^{[0]} & =\frac{\delta^{2}}{3 q^{4}}\left[\left(\lambda_{1}\right)^{4}-\left(\lambda_{2}\right)^{4}\right],
\end{aligned}
$$

where

$$
\lambda_{1}=\ln \left(z_{1}\right), \quad \lambda_{2}=\ln \left(z_{2}\right), \quad z_{2}=\xi_{0} / \delta .
$$

Several interesting facts emerge from (7.6) and Fig. 5: (a) the mean FPT does not coincide with the $e$-folding timescale [see (7.2)]; (b) the mean FPT increases with decreasing $\delta$ logarithmically as $-\ln \left(\delta^{2}\right)$; (c) the variance of FPT (proportional to $\delta^{2}$ ) decreases with decreasing $\delta^{2}$. Therefore, the reduction of the noise level only decreases the variance of FPT considerably, but not the mean FPT.

\section{2) NonLINEAR REgime}

For nonlinear regime $(g \neq 0)$, the mean FPT is obtained using the iterative method illustrated in appendix A:

$$
\langle\tau\rangle \approx\langle\tau\rangle^{[0]}+\frac{1}{3 q^{2}}\left(R^{[1]}+R^{[2]}+R^{[3]}+R^{[4]}\right),
$$

where 


$$
\begin{aligned}
& R^{[1]}=B_{1} \frac{g \delta}{q^{2}}\left[\left(\lambda_{1}\right)^{3}-\left(\lambda_{2}\right)^{3}\right], \\
& R^{[2]}=B_{2} \frac{g^{2} \delta^{2}}{q_{4}}\left[\left(\lambda_{1}\right)^{4}-\left(\lambda_{2}\right)^{4}\right], \\
& R^{[3]}=B_{3} \frac{g^{3} \delta^{3}}{q^{6}}\left[\left(\lambda_{1}\right)^{5}-\left(\lambda_{2}\right)^{5}\right], \\
& R^{[4]}=B_{4} \frac{g^{4} \delta^{4}}{q^{8}}\left[\left(\lambda_{1}\right)^{6}-\left(\lambda_{2}\right)^{6}\right],
\end{aligned}
$$

and the coefficients $B_{1}, B_{2}, B_{3}$, and $B_{4}$ are given by

$$
\begin{aligned}
& B_{1}=2.66 ; \quad B_{2}=1.33 ; \quad B_{3}=0.36 ; \\
& B_{4}=0.04 .
\end{aligned}
$$

Figure 5 shows the comparison of the mean and variance of FPT between linear and nonlinear regimes with three different tolerance levels $(\varepsilon=0.01,0.1$, and 0.5$)$. For the small tolerance level $(\varepsilon=0.01)$ the nonlinear effect on both the mean and variance of FPT is negligible. For $0.01<\varepsilon<0.1$, the nonlinear effect is negligible on the mean FPT too but not on the variance of FPT. For $\varepsilon=0.5$, the prediction skill difference between the tangent and nonlinear error models becomes very evident. For the same initial error, the mean FPT is larger for the nonlinear regime than for the linear regime, which indicates the enhancement of the predictability due to nonlinearity.

\section{c. High predictability $(\lambda<1)$}

As $\lambda<1$, the mean and variance of FPT increase with decreasing $\lambda$ rapidly (Fig. 4), which leads to the high-predictability range.

\section{1) LinEAR REGIME $(g=0)$}

For $\lambda \rightarrow 1$, the mean and variance of FPT are given by

$$
\begin{gathered}
\langle\tau\rangle^{[0]} \approx \frac{2}{(1-\lambda)^{2} q^{2}}\left(z_{1}\right)^{1-\lambda}\left[1-(z)^{\lambda-1}\right] \text { and } \\
\left\langle\delta \tau^{2}\right\rangle^{[0]} \approx \frac{4}{q^{4}(1-\lambda)^{4}}\left(z_{1}\right)^{2(1-\lambda)}\left(1-z^{2(\lambda-1)}\right) .
\end{gathered}
$$

Equation (7.9) demonstrates that the mean FPT does not coincide with the $e$-folding timescale (7.2) even for infinitesimal and small amplitude errors. In addition it is noted that for high predictability and $z \gg$ 1 , the mean and variance of FPT have power dependence of $z_{1}$ with scaling exponents equaled to $1-\lambda$ and $2(1-\lambda)$, respectively.

\section{2) Nonlinear REGiMe $(g \neq 0)$}

For the nonlinear regime, the mean and variance of FPT are given by

$$
\langle\tau\rangle=\langle\tau\rangle^{[0]}+\frac{4 g \varepsilon}{3 q^{2}(1-\lambda)}\left(R^{[1]}+R^{[2]}+R^{[3]}+R^{[4]}\right),
$$

where

$$
\begin{aligned}
& R^{[1]}=C_{1} \frac{1}{q^{2}}\left(1-z^{2(1-\lambda)}\right), \quad R^{[2]}=C_{2} \frac{g \varepsilon}{q^{3}}\left(1-z^{3(1-\lambda)}\right), \\
& R^{[3]}=C_{3} \frac{g^{2} \varepsilon^{2}}{q^{4}}\left(1-z^{4(1-\lambda)}\right), \\
& R^{[4]}=C_{4} \frac{g^{3} \varepsilon^{3}}{q^{5}}\left(1-z^{5(1-\lambda)}\right),
\end{aligned}
$$

and $C_{1}, C_{2}, C_{3}$, and $C_{4}$ are the coefficients given by

$$
\begin{aligned}
C_{1} & =\frac{1}{2-\lambda} ; \quad C_{2}=\frac{3}{(3-\lambda)} ; \quad C_{3}=\frac{4}{9(4-\lambda)} ; \\
C_{4} & =\frac{32}{135(5-\lambda)}
\end{aligned}
$$

For $\lambda=0.5$, they are

$$
C_{1}=0.67, \quad C_{2}=1.2, \quad C_{3}=0.13, \quad C_{4}=0.05,
$$

which indicates a slower convergence of the iteration process (reduction values from $C_{1}$ to $C_{4}$ ) than in the low-predictability regime $(\lambda \gg 1)$ represented by the reduction values from $A_{1}$ to $A_{4}$.

Figure 6 shows the comparison of the mean and variance of FPT between the linear and nonlinear error models for three different tolerance levels $(\varepsilon=$ $0.01,0.1$, and 0.5$)$. For the small tolerance level $(\varepsilon$ $=0.01)$, the nonlinear effect on both mean and variance of FPT is negligible. As the tolerance level increases (i.e., $\varepsilon>0.01$ ), the mean FPT is larger in the nonlinear regime than in the linear regime for the same initial condition error vector, which indicates the enhancement of the predictability due to nonlinearity. Besides, both solid and dotted curves in Fig. 6 reveal a reverse $\Gamma$-type shape, which indicates that $\langle\tau\rangle$ and $\left\langle\delta \tau^{2}\right\rangle$ depend weakly on the value of initial error in both linear and nonlinear regimes if $\xi_{0} / \varepsilon \ll$ 0.01 .

\section{Ensemble effect of initial uncertainty on the mean FPT}

The error of an ensemble forecast is usually smaller than the mean error of individual forecasts comprising the ensemble (Leith 1974; Murthy 1991). One may ask: What is the ensemble effect of initial uncertainty with finite amplitudes on the prediction skill? The classical stability analysis associated with Lyapunov exponents or SVs is not able to answer this question since it is in general only for small initial errors. The FPT approach can answer this question, and the low predictability range $(\lambda>1)$ is taken as the example for illustration. To do so, let the initial condition be distorted by noises with unknown intensity 

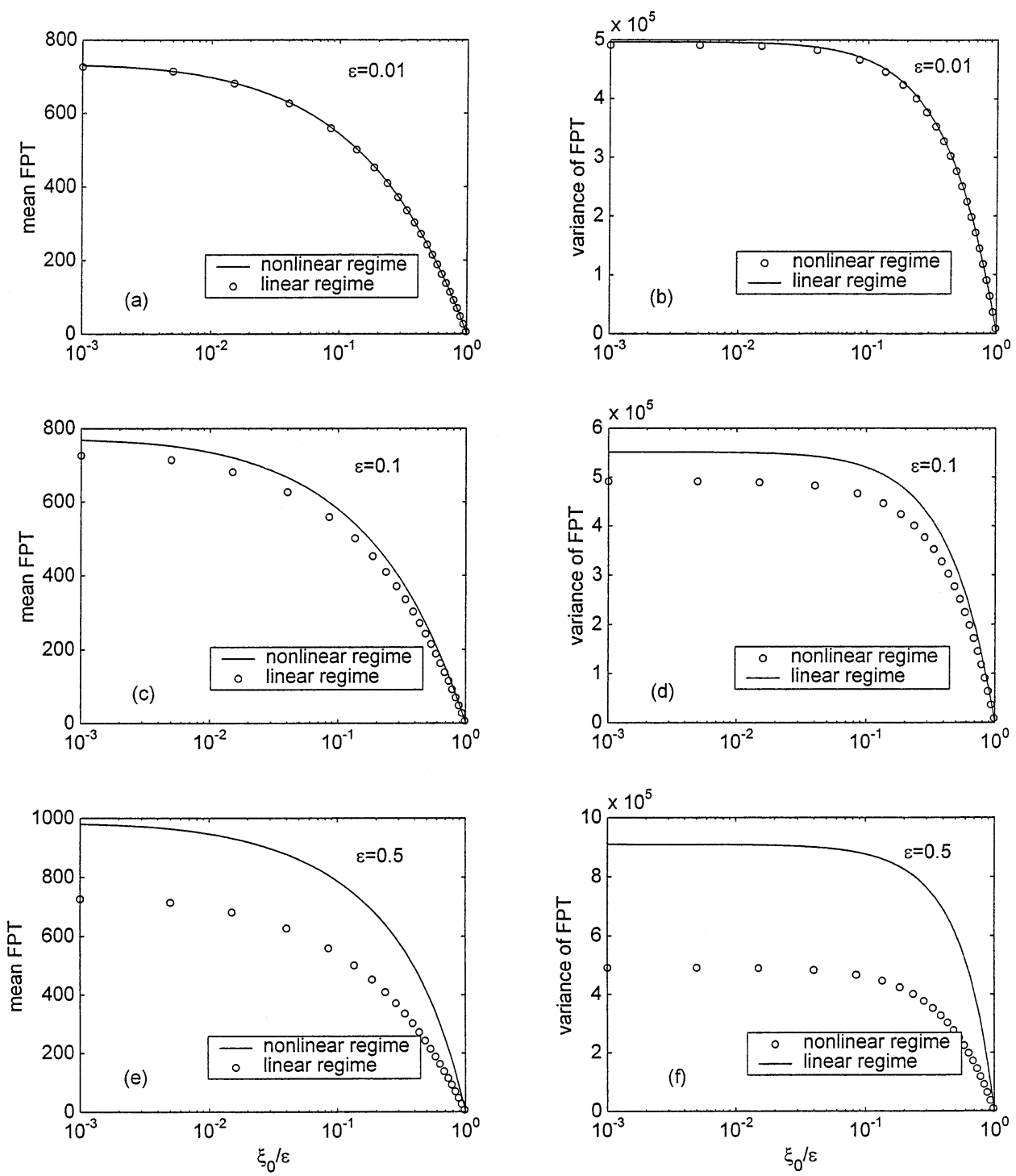

FIG. 6. Same as Fig. 5, except for $\lambda=0.5$.

of $\hat{\xi}_{0}$. According to Leith (1974) additional noises with zero mean and variance of $4 \tilde{\xi}^{2}$ are added to $\hat{\xi}_{0}$. The mean FPT is averaged over ensemble realizations composed of individual samples with noises homogeneously distributed within the interval $\left[\hat{\xi}_{0}-\tilde{\xi}, \hat{\xi}_{0}+\tilde{\xi}\right]$.

\section{a. Infinitesimal and small initial errors}

Independent of the tolerance level, we obtain the following expression:

$$
\left\langle\left\langle\tau\left(\hat{\xi}_{0}\right)\right\rangle\right\rangle>\left\langle\tau\left(\hat{\xi}_{0}\right)\right\rangle,
$$

for infinitesimal and small initial errors. Here, the single bracket indicates an averaging over statistics of stochastic forcing and the double bracket denotes an averaging operator over statistics of both stochastic forcing and the ensemble of initial realization. The mathematical detail is listed in appendix B. The inequality (8.1) shows that the ensemble mean of forecasts will, on average, have longer FPT (smaller model error) than the mean error of individual forecast comprising the ensemble. This agrees with the classical result (Leith 1974; Murphy 1991).

\section{b. Finite amplitude initial errors}

For finite amplitude initial errors, the ensemble mean FPT may be smaller than the mean FPT for an individual prediction. Therefore, if the initial uncertainty consists of small and finite amplitude errors and if the tolerance level is large, the additional averaging over the ensemble of the initial errors may deteriorate the model prediction skill. 


\section{Conclusions}

This study demonstrates the capability of the FPT approach in evaluating model forecast skill. Two kinds of predictability skill are analytically identified for the Lorenz (1984a) model based on the parameter, $\lambda=2 \sigma /$ $q^{2}$ : low predictability if $\lambda>1$, and high predictability if $\lambda<1$. Here, $\sigma$ and $q$ are the maximum Lyapunov exponent and the intensity of attractor fluctuations, respectively. The low- to high-predictability transition occurs at $\lambda=1$. The transit from low to high predictability is realized for $\lambda=1$. Both kinds of predictability skill coexist for small initial error $\xi_{0} \ll 1$.

The low predictability $(\lambda>1)$ is characterized by high sensitivity to initial condition errors. For a small tolerance level $(\varepsilon \ll 1)$, the model prediction skill is represented by the $e$-folding time. Both the mean and variance of FPT increase with increasing intensity of the attractor fluctuations. The mean FPT is longer in the nonlinear than in the linear regimes. The model prediction skill should weakly depend on subgrid-scale parameterization schemes; however, neglect of the noise scale $(\delta=0)$ leads to an incorrect asymptote for the mean FPT when the initial error is infinitesimal. For a large tolerance level, the mean FPT is no longer exponentially decaying with the initial error, and is always greater than the $e$-folding time.

The high predictability $(\lambda<1)$ is characterized by the power-law decay of the mean and variance of FPT with decay exponents $(1-\lambda)$ and $2(1-\lambda)$, respectively, if $z \gg 1$ and $\lambda \rightarrow 1$. The prediction skill strongly depends on the noise level $\delta$ and the subgrid-scale parameterization schemes. Notice that both the mean and variance of FPT weakly depend on the values of infinitesimal initial errors.

A nonlinear perspective of forecast error is important in finding an exact functional correlation between the quantitative model skill and the physical factors. In general, nonlinear processes limit the growth of forecast error and causes a wide dispersion of individual predictions. Averaging over the ensemble of initial uncertainties, the nonlinearity can either increase or decrease the mean FPT.

The model prediction skill strongly depends on the position of the predicted attractor. Low- or high- (local) prediction skill should be locally detected through PDP analysis on different fragments of the attractor trajectory. However, the real model skill is obtained through averaging over the local prediction skills along the reference trajectory. The high (low) predictability can be interrupted by randomly distributed bursts of low (high) predictability. Such a temporal intermittency of the prediction skill should be investigated in more complete model than the Nicolis model. This is not discussed in this paper.

Acknowledgments. This work was supported by the Office of Naval Research (ONR) Naval Ocean Mod- eling Prediction (NOMP) program, Naval Oceanographic Office, and the Naval Postgraduate School. L. Ivanov thanks the National Research Council for an Associateship Award at the Naval Postgraduate School. T. Margolina and O. Melnichenko were supported by U.S. Civil Research and Development Foundation through the Grant UG 2079. We are very grateful to Prof. C. Nicolis and a second anonymous reviewer for useful comments.

\section{APPENDIX A}

\section{Iterative Process for Solving (5.5) and (5.6)}

Taking (5.6) as an example, the iterative process starts from the decomposition of the operator

$$
\hat{L}=\left(\sigma \xi_{0}-g \xi_{0}^{2}\right) \frac{d}{d \xi_{0}}+\frac{q^{2} \xi_{0}^{2}}{2} \frac{d^{2}}{d \xi_{0}^{2}}
$$

into two parts

$$
\hat{L}=L_{1}+\mu L_{2}
$$

where $\mu$ is the varying parameter ranged from 0 to 1 . The operator $L_{1}$ is chosen such that the equation

$$
L_{1} \tau=-1
$$

has an analytical solution.

Then, we introduce an additional auxiliary function $W=\partial \tau / \partial \mu$ satisfying the following equation:

$$
L_{1} W+\mu L_{2} W+L_{2}\langle\tau\rangle=0 .
$$

The following iterative process is used to solve (A.4) (Ivanov et al. 1999):

$$
\begin{gathered}
L_{1}\langle\tau\rangle^{[0]}=-1, \\
\langle\tau\rangle^{[i]}=\langle\tau\rangle^{[i-1]}+\Delta \mu W^{[i-1]}, \\
i=1, \ldots, I \\
L_{1} W^{[i]}+L_{2}\langle\tau\rangle^{[i]}+\mu^{[i]} L_{2} W^{[i-1]}=0, \\
\mu^{[i]}=\Delta \mu i, \quad \Delta \mu=\frac{1}{I},
\end{gathered}
$$

where $I$ is the maximum number of iterations. The iteration stops when $\mu^{[i]}$ equals 1 . Here, $\langle\tau\rangle^{[i]}$ is the approximate solution of (5.5) with accuracy of $O(\Delta \mu)$. The variance of FPT is calculated using the same iterative procedure.

Let us illustrate the iterative procedure (A.5)-(A.8) by calculating the mean FPT for $\lambda=1$ and $I=3$ : 


$$
\begin{aligned}
L_{1}\langle\tau\rangle^{[0]}= & -1 \\
L_{1} W^{[0]}= & -L_{2}\langle\tau\rangle^{[0]} \\
L_{1} W^{[1]}= & -L_{2}\langle\tau\rangle^{[0]}-\left(\mu^{[1]}+\Delta \mu\right) L_{2} W^{[0]}, \\
L_{1} W^{[2]}= & -L_{2}\langle\tau\rangle^{[0]}-\Delta \mu L_{2} W^{[0]} \\
& -\left(\mu^{[2]}+\Delta \mu\right) L_{2} W^{[1]} \\
L_{1} W^{[3]}= & -L_{2}\langle\tau\rangle^{[0]}-\Delta \mu L_{2} W^{[0]}-\Delta \mu L_{2} W^{[1]} \\
& -\left(\mu^{[3]}+\Delta \mu\right) L_{2} W^{[2]}
\end{aligned}
$$

where Eqs. (A.9)-(A.13) use the reflecting boundary condition at $\xi_{0}=\delta$ and the absorbing boundary condition at $\xi_{0}=\varepsilon$, respectively. The solutions are written by

$$
\begin{aligned}
\langle\tau\rangle^{[0]}= & \frac{1}{q^{2}} R\left(\frac{\varepsilon}{\delta}, \frac{\xi_{0}}{\delta}, 2\right), \\
W^{[0]}= & \frac{2}{3 q^{2}} B_{1} R\left(\frac{\varepsilon}{\delta}, \frac{\xi_{0}}{\delta}, 3\right), \\
W^{[1]}= & \frac{2}{3 q^{2}} B_{1} R\left(\frac{\varepsilon}{\delta}, \frac{\xi_{0}}{\delta}, 3\right)+\frac{2}{9 q^{2}} B_{2} \Delta \mu_{1} R\left(\frac{\varepsilon}{\delta}, \frac{\xi_{0}}{\delta}, 4\right), \\
W^{[2]}= & \frac{2}{3 q^{2}} B_{1} R\left(\frac{\varepsilon}{\delta}, \frac{\xi_{0}}{\delta}, 3\right)+\frac{4}{9 q^{2}} B_{2} \Delta \mu_{1} R\left(\frac{\varepsilon}{\delta}, \frac{\xi_{0}}{\delta}, 4\right) \\
& +\frac{4}{45 q^{2}} B_{3} R\left(\frac{\varepsilon}{\delta}, \frac{\xi_{0}}{\delta}, 5\right), \\
W^{[3]}= & \frac{2}{3 q^{2}} B_{1} R\left(\frac{\varepsilon}{\delta}, \frac{\xi_{0}}{\delta}, 3\right)+\frac{2}{3 q^{2}} B_{2} \Delta \mu_{1} R\left(\frac{\varepsilon}{\delta}, \frac{\xi_{0}}{\delta}, 4\right) \\
& +\frac{36}{135 q^{2}} B_{3} R\left(\frac{\varepsilon}{\delta}, \frac{\xi_{0}}{\delta}, 5\right)+\frac{16}{405 q^{2}} B_{4} R\left(\frac{\varepsilon}{\delta}, \frac{\xi_{0}}{\delta}, 6\right),
\end{aligned}
$$

where

$$
\begin{gathered}
R\left(z_{1}, z_{2}, l\right)=\ln ^{l} z_{1}-\ln ^{l} z_{2}, \quad B_{n}=\left(\frac{g \delta}{q^{2}}\right)^{n}, \\
n=1, \ldots, 4 .
\end{gathered}
$$

\section{APPENDIX B}

\section{Averaging over Initial Uncertainty}

a. Small amplitude error and small tolerance level Let

$$
\begin{aligned}
\xi_{0}^{\min } & =\hat{\xi}_{0}-\tilde{\xi}, & \xi_{0}^{\max } & =\hat{\xi}_{0}+\tilde{\xi}, \\
\beta & =\xi_{0}^{\min } / \xi_{0}^{\max }, & \hat{\xi}_{0} & =0.5\left(\xi_{0}^{\max }+\xi_{0}^{\min }\right) .
\end{aligned}
$$

Average of (7.2) over an ensemble of initial uncertainty is given by

$$
\begin{aligned}
\langle\tau\rangle=\frac{1}{q^{2}(\gamma-1)}[ & \ln \left(\frac{\varepsilon}{\xi_{0}^{\max }}\right)+1 \\
& \left.+\frac{\xi_{0}^{\min }}{\xi_{0}^{\max }-\xi_{0}^{\min }} \ln \left(\frac{\xi_{0}^{\min }}{\xi_{0}^{\max }}\right)\right] .
\end{aligned}
$$

Rewrite (B.1) and (7.2) into

$$
\begin{gathered}
\left\langle\left\langle\tau\left(\hat{\xi}_{0}\right)\right\rangle\right\rangle=\frac{2}{q^{2}(\gamma-1)}\left[\ln \left(\frac{\varepsilon}{\xi_{0}^{\max }}\right)+1+\frac{\beta}{1-\beta} \ln (\beta)\right], \\
\left\langle\tau\left(\hat{\xi}_{0}\right)\right\rangle=\frac{2}{q^{2}(\gamma-1)}\left[\ln \left(\frac{\varepsilon}{\xi_{0}^{\max }}\right)+\ln \left(\frac{2}{1+\beta}\right)\right] . \quad \text { (B.3) }
\end{gathered}
$$

A comparison between (B.3) and (B.4) leads to

$$
\left\langle\left\langle\tau\left(\hat{\xi}_{0}\right)\right\rangle\right\rangle>\left\langle\tau\left(\hat{\xi}_{0}\right)\right\rangle \text { if } \beta \leq 0.9 .
$$

\section{b. Small amplitude error and large tolerance level}

For this case, $\xi_{0} / \varepsilon \ll 1$, a constant $A$,

$$
A=\frac{4}{3 q^{2}(\lambda-1)}\left[\frac{g \varepsilon}{q^{2}}+\frac{g^{2} \varepsilon^{2}}{q^{4}}+\frac{g^{3} \varepsilon^{3}}{q^{6}}+\frac{g^{4} \varepsilon^{4}}{q^{8}}\right]
$$

is added to both (B.2) and (B.3). The analysis becomes the same as the previous case (i.e., the small amplitude error and small tolerance level).

\section{c. Large amplitude error and large tolerance level}

Use of the asymptotic

$$
\ln \left(\frac{\varepsilon}{\xi_{0}}\right) \approx \frac{\varepsilon}{\xi_{0}}-1 \quad \text { as } \frac{\varepsilon}{\xi_{0}} \rightarrow 1,
$$

and average of all terms of (7.4) over the ensemble of initial errors leads to

$$
\begin{gathered}
\left\langle\tau\left(\hat{\xi}_{0}\right)\right\rangle=\frac{1}{q^{2}(\gamma-1)}\left\{G+\frac{4 g^{2} \varepsilon^{2} A_{2}}{3 q^{4}}\left[1-\frac{1}{4} \frac{\xi_{\max }^{2}}{\varepsilon^{2}}\left(1+2 \beta+\beta^{2}\right)\right]+\frac{4 g^{2} \varepsilon^{2} A_{3}}{3 q^{6}}\left[1-\frac{\hat{\xi}_{0}}{4 \varepsilon^{2}}\left(1+2 \beta+\beta^{2}\right)\right]+\cdots\right\}, \\
\left\langle\left\langle\tau\left(\hat{\xi}_{0}\right)\right\rangle\right\rangle=\frac{1}{q^{2}(\gamma-1)}\left\{G+\frac{4 g^{2} \varepsilon^{2} A_{2}}{3 q^{4}}\left[1-\frac{1}{3} \frac{\xi_{\max }^{2}}{\varepsilon^{2}}\left(1+\beta+\beta^{2}\right)\right]+\frac{4 g^{3} \varepsilon^{3} A_{3}}{3 q^{6}}\left[1-\frac{\hat{\xi}_{0}}{2 \varepsilon^{2}}\left(1+\beta^{2}\right)\right]+\cdots\right\},
\end{gathered}
$$


where

$$
G=\left(-1+\frac{4 g \varepsilon A_{1}}{3 q^{2}}\right)\left(1-\frac{\hat{\xi}_{0}}{2 \varepsilon}\right) .
$$

Comparison between (B.4) and (B.5) leads to

$$
\left\langle\left\langle\tau\left(\hat{\xi}_{0}\right)\right\rangle\right\rangle\left\langle\left\langle\tau\left(\hat{\xi}_{0}\right)\right\rangle\right.
$$

for any value of $\beta$.

\section{REFERENCES}

Anderson, J. L., and H. M. van der Dool, 1994: Skill and return skill in dynamic extended range forecasts. Mon. Wea. Rev., 122, 507516.

Aurell, E., G. Boffetta, A. Crisanti, G. Paladin, and A. Vulpiani, 1996: Growth of noninfinitesimal perturbations in turbulence. Phys. Rev. Lett., 77, 1263-1265.

Barkmeijer, J., 1996: Constructing fast-growing perturbations for the nonlinear regime. J. Atmos. Sci., 53, 2838-2851.

Benzi, R., and G. F. Carnevale, 1989: A possible measure of local predictability. J. Atmos. Sci., 46, 3593-3598.

_ , A. Mazzino, M. Marrocu, and E. Trovatore, 1999: Character ization of the long-time and short-time predictability of loworder models of the atmosphere. J. Atmos. Sci., 56, 3495-3507.

Boffetta, G., P. Giuliani, G. Paladin, and A. Vulpiani, 1998: An extension of the Lyapunov analysis for the predictability problem. J. Atmos. Sci., 55, 3409-3416.

Chu, P. C., 1999: Two kinds of predictability in the Lorenz system. J. Atmos. Sci., 56, 1427-1432.

—, L. M. Ivanov, and C. W. Fan, 2002: Backward Fokker-Planck equation for determining model valid prediction period. J. Geophy. Res., in press.

Dalcher, A., and E. Kalnay, 1987: Error growth and predictability in operational ECMWF forecasts. Tellus, 39A, 474-491.

Ehrendorfer, M., 1994a: The Liouville equation and its potential usefulness for the prediction of forecast skill. Part I: Theory. Mon Wea. Rev., 122, 703-713.

_ 1994b: The Liouville equation and its potential usefulness for the prediction of forecast skill. Part II: Applications. Mon. Wea. Rev., 122, 714-728.

Farrell, B. F., and P. J. Ioannou, 1996a: Generalized stability theory. Part I: Autonomous operations. J. Atmos. Sci., 53, 2025-2040.

_ $1996 \mathrm{~b}$ : Generalized stability theory. Part II: Nonautonomous operations. J. Atmos. Sci., 53, 2041-2053.

Gardiner, C. W., 1985: Handbook of Stochastic Methods for Physics, Chemistry and the Natural Sciences. Springer-Verlag, 526 pp.

Gustafsson, N., E. Kallen, and S. Thorsteinsson, 1998: Sensitivity of forecast errors to initial and lateral boundary conditions. Tellus, 50A, 176-185.

Has'minskii, R. Z., 1980: Stochastic Stability of Differential Equations. Sijthoff and Noordhoff, $341 \mathrm{pp}$.
Ivanov, L. M., and T. M. Margolina, 1999: Prediction in geophysical hydrodynamics as the probabilistic problem of the first-passage boundary. Proc. Rossby-1000 Symp., Stockholm Sweden, Dept. of Meteorology, Stockholm University, 63-69.

- A. D. Kirwan Jr., and O. V. Melnichenko, 1994: Prediction of the stochastic behavior of nonlinear systems by deterministic models as a classical time-passage probabilistic problem. Non. Proc. Geophys., 1, 224-233.

_ T. M. Margolina, and O. V. Melnichenko, 1999: Prediction and management of extreme events based on a simple probabilistic model of the first-passage boundary. Phys. Chem. Earth, 24A, 169-173.

Jiang, S., and P. Malanotte-Rizzoli, 1999: On the predictability of regional oceanic jet stream: The impact of model errors at the inflow boundary. J. Mar. Res., 57, 641-669.

Leith, C. E., 1974: Theoretical skill of Monte Carlo forecasts. Mon. Wea. Rev., 102, 409-418.

_- 1996: Stochastic models of chaotic systems. Physica D, 98, 481-491.

Lorenz, E. N., 1984a: Irregularity. A fundamental property of the atmosphere. Tellus, 36A, 98-110.

_ 1984b: The local structure of a chaotic attractor in four dimensions. Physica D, 13, 90-104

Molteni, F., and S. Corti, 1998: Long-term fluctuations in the statistical properties of low-frequency variability: Dynamical origin and predictability. Quart. J. Roy. Meteor. Soc., 124, 495-526.

Murphy, A. H., 1991: Forecast verification: Its complexity and dimensionality. Mon. Wea. Rev., 119, 208-219.

Nicolis, C., 1992: Probabilistic aspects of error growth in atmospheric dynamics. Quart. J. Roy. Meteor. Soc., 118, 553-568.

Palmer, T. N., 2001: A nonlinear dynamical perspective on model error: A proposal for non-local stochastic-dynamic parameterization in weather and climate prediction models. Quart. J. Roy. Meteor. Soc., 127, 279-304.

Penland, C., and P. D. Sardeshmukh, 1995: The optimal growth of tropical sea surface temperature anomalies. J. Climate, 8, 19992024.

Pontryagin, L. S., V. G. Boltyanskii, R. V. Gamkrelidze, and E. F. Mishenko, 1969: Mathematical Theory of Optimal Process (in Russian). 2d ed. M. Nauka, 384 pp. [Also available as, 1962: The Mathematical Theory of Optimal Processes. (Translation from the 1 st ed.) InterScience, $260 \mathrm{pp}$.]

Press, W. H., S. A. Teukolsky, W. T. Vetterling, and B. P. Flannery, 1992: Numerical Recipes in FORTRAN: The Art of Scientific Computing. 2d ed. Cambridge University Press, 963 pp.

Toth, Z., and E. Kalnay, 1997: Ensemble forecasting at NCEP and the breeding method. Mon. Wea. Rev., 125, 3297-33109.

Vannitsem, S., and C. Nicolis, 1997: Lyapunov vectors and error growth patterns in a T21L3 quasi-geostrophic model. J. Atmos. Sci., 54, 347-361.

Vukicevic, T., 1991: Nonlinear and linear evolution of initial forecast errors. Mon. Wea. Rev., 119, 1602-1611.

Ziehmann, C., L. A. Smith, and J. Kurths, 2000: Localized Lyapunov exponents and the prediction of predictability. Phys. Lett., A271, 237-251. 